\title{
THE ROLE OF WATER ENVIRONMENT REHABILITATION IN PATIENTS WITH NEUROLOGICAL AND COGNITIVE DISABILITIES
}

\author{
Barassi G. ${ }^{1}$, Bellomo R.G. ${ }^{2}$, Ancona E. ${ }^{3}$, Trivisano L. ${ }^{4}$, Saggini R. ${ }^{5}$ \\ ${ }^{1}$ Faculty of Physiotherapy, "G. d'Annunzio" University of Chieti-Pescara, ${ }^{2}$ Physical and Rehabilitation \\ Medicine, "G. d'Annunzio" University of Chieti-Pescara, ${ }^{3}$ Postgraduate school in Physical and Rehabilitation \\ Medicine, "G. d'Annunzio" University of Chieti-Pescara, ${ }^{4}$ Rehabilitation Department, Local Health Unit of \\ Foggia, ${ }^{5}$ Physical and Rehabilitation Medicine, "G. d'Annunzio" University ofChieti-Pescara
}

Key words: Water therapy, water rehabilitation, myofascial manual therapy, trigger point, neurological disability, cognitive disability

\begin{abstract}
:
The interaction between the body system and a microgravity environment influences the biomechanical behaviors allowing the reorganization of sensorimotor relations through "unique" stimulation of the functional hierarchies of the central nervous system, thus changing the motor gesture as a whole. The physical characteristics of the aquatic environment can be exploited by the rehabilitative operator to achieve specific muscle, neurological and sensory recovery with different modality and times compared to the techniques used in a dry environment, stimulating the re-acquisition of neuromotor-sensory skills impaired or even lost. The purpose of the study is to evaluate the effectiveness of a rehabilitation protocol in the thermal aquatic environment, on postural parameters, gait, balance, and autonomy on the disabled patient. 50 subjects (mean age 48.5 years) were evaluated and treated because of the suffering of neuromotor cognitive and/or mental disabilities. Subjects underwent two evaluations, before and after a rehabilitation protocol in aquatic environment, consisting of 20 therapy sessions in thermal water, made of a physiatric specialistic examination, administration of FIM and Tinetti SCALE and Computerized Postural assessment without markers (Sa.BB Imaging), in order to assess the effects of the protocol on the health status of the patient, evaluating the ADL, balance and postural parameters and if these three data show a correlation in clinical practice. Considering the scores of the FIM scale, neurological patients went from a score of 84,323 to 97,29 after 20 sessions of treatment $(\mathrm{p}=0.0487)$. Patients with mental disabilities increased from a score of 76,895 to $94,63(\mathrm{p}=$ 0.0383). In all patients the FIM scale score increased from 82,692 to 95,75 ( $p=0.0107)$. Gait and balance, evaluated through the Tinetti scale, improved from 20,105 to 24,474 ( $\mathrm{p}=0.04)$ for patients with mental disabilities and from 11,774 to $13,742(\mathrm{p}=0.4688)$ for patients with neuromotor disabilities. In all patients the Tinetti score rose from 13,385 to 17,885 ( $p=0.4688$ ). These data were validated by the improvements achieved at the postural level, particularly in the values concerning the anteposition of the head, important for the distribution of the load in the polygon of support; also supported by the reduction of the lateral side oscillations of the pelvis. We can conclude that the technique used resulted in a great increase of postural and functional parameters of the subjects of the study.
\end{abstract}

\section{INTRODUCTION:}

\subsection{Role of the aquatic environment rehabilitation}

It is actually well known how the aquatic environment can play an important role during a rehabilitation phase, thanks to its unique physical characteristics (including reduction of the gravity load on joints and body relaxation through modifications of body's temperature). This occurs because of the interaction between the body system, and a microgravity environment

Submitted September 13, 2016 / Accepted for publication March 15, 2017 
influences the biomechanical behaviors of the subject allowing a reorganization of sensorimotor relations through "unique" stimulation of the functional hierarchies of the central nervous system, thus changing the motor gesture as a whole. The physical characteristics of the aquatic environment can be exploited by the rehabilitative operator to achieve specific muscle that require treatment finalized to a neurological and sensory recovery of the patient with different modality and times compared to the techniques used in dry environment, stimulating the reacquisition of neuromotor-sensory skills impaired or even lost as a consequence of the pathology ${ }^{1) 2}$.

\subsection{Effects of the aquatic environment on the human body: the posture and the myofascial syndrome}

The fundamental premise to establish how the microgravity aquatic environment produces its effects on the human body is to consider the body system as a cybernetic set of structure functionally correlated and operating with the common purpose to realize movements in compliance with laws of balance and energetic economy in the absence of pain finalized aimed at achieving what we might call "ideal posture"4). In this context, some experimental data" demonstrated how the re-weighting mechanism of postural subsystems contributes not only to achieving a good statical control but also good dynamic attitudes. The integration of these postural subsystems is functionally realized by the connective tissue, defined as a fibrous system connecting all parts of the human body ${ }^{5,6)}$. The connective tissue plays, in fact, a lot of roles in the body, taking part in various physio-biological pathways (acid-base balance, hydric-saline balance, blood flow and nervous impulses conduction). It's role in the regulation of posture is determined by the great amount of proprioceptors (in particular Golgi tendon organs and neuromuscular fuses) and esteroceptors paired with its physical actions of structuration and separation of muscles. This can be summarized in the realization of a complex system composed of active kinetic myofascial chains that interface with the surrounding environment in a continuous stream of information and adaptations. Assuming that the connective fascia can be damaged by a lot of factors correlated to the numerous function covered by this tissue, it is also possible to talk about muscular, or better myofascial dysfunctions consisting of spasms and states of excessive tension determined by restrictions of the fascia. According to the actual definition of myofascial syndrome ${ }^{2), 3)}$ we can detect the main dysfunctional area in a muscle, at which we can refer to as trigger point. A trigger point is substantially the area of maximal retraction of the myofascial system which usually generates symptoms like local and referred pain, reduction of the articular range of motion and postural compensations which can alter the health state of a subject.

\subsection{Fields of application: patients}

In this wide rehabilitative context it is important to assess a rehabilitative method able to restore a functional homeostatic balance of a patient, exploiting all the actual knowledge about the gold standard in rehabilitation such as aquatic environment treatments and manual therapies. For all these reasons our work focuses on patients suffering from neuromotor cognitive and/or mental disabilities coming from a home-care kind rehabilitation, which was suspended during the execution of our study.

\section{AIM:}

The aim of our study is to assess if a specific algo-ostheo-myofascial technique, that we named Sa.B.B.(Safe Bearing Back), realized in a microgravity water environment can be successfully used in the rehabilitation of patient with various kind of pathologies, rebalancing both the static posture of patients and the dynamic abilities in the execution of the activities of daily living. The expected effect of the protocol should be attributed to the interaction between the patient and the operator by the application of specific manual techniques and mobilizations realized in a thermal water environment. 


\section{MATERIALS AND METHODS:}

\subsection{Presentation of the study: ethic and design}

This experimental study and the research protocol is compatible with the WMA's Declaration of Helsinki. and all subjects involved in the study have been informed about the procedure and the aim of the research and signed the informed consent.

This study has been carried out in the context of the Chair of Physical and Rehabilitative Medicine of the "G. D'Annunzio" University of Chieti-Pescara, director Raoul Saggini, and realized in the Thermal Rehabilitation Center of Castelnuovo della Daunia (Foggia), Italy.

The thermal aquatic center has been selected because of the characteristics of the water, in particular its mineral bicarbonate-sulphate-alkaline-earthy characteristics used in rehabilitative and functional sub-aqueous modality.

\subsection{Therapeutical approach: the Sa.B.B. Technique}

The therapeutical approach selected for the treatment in the microgravity environment is named Sa.B.B. (Safe Bearing Back) technique. This is an algo-ostheo-myofascial technique based on the principles of biomechanical loading of soft tissues and modification of neural reflexes through a specific receptorial stimulation ${ }^{13}$.

This modification is reached through extra sensory inputs given to specific body's areas of the patient regarding the spatial orientation of its segments. This allows to eliminate a typical overload of visual and auditory perceptive channels while restoring a good control of other proprioceptive channels especially through the application of vibrotactile and electrotactile stimuli, according to some researches made during the $90{ }^{\prime} \mathrm{s}^{7,8}$.

\subsection{The sample and evaluation methods}

To test the therapeutic potential of the Sa.B.B. technique 50 subjects were evaluated and treated because suffering from neuromotor cognitive and/or mental disabilities. The main inclusion criteria was represented by a minimum disability score of $67 \%$, according to the Italian National Health Service evaluation criteria, due to conditions such as stroke, spinal lesions, cerebral palsy and so on. Exclusion criteria were mainly the presence of other pathologies, like cardiovascular diseases, open wounds, infective processes, rheumatic pathologies and severe cancer. All patients came from a traditional kind of neurological rehabilitation consisting in passive mobilizations, Bobath method techniques such as Reflex Inhibiting Patterns, Perfetti method therapeutic exercises, and classical neuromotor exercise training. This previous rehabilitation program was suspended while patients performed our study. Subjects underwent two evaluations, one before the rehabilitation protocol realized in the thermal microgravity aquatic environment (T0) and one at the end of the 20 therapy sessions (T1). These evaluations consisted of a physiatric examination, the administration of the FIM and Tinetti Scale and a Computerized Postural analysis without markers realized by with the Sa.BB Imaging software. The main goal of this study was to assess the effects of the protocol on the general health state of the patient, evaluating qualitative changes of ADL and balance and postural parameters and also if these three data showed a correlation in clinical practice. 

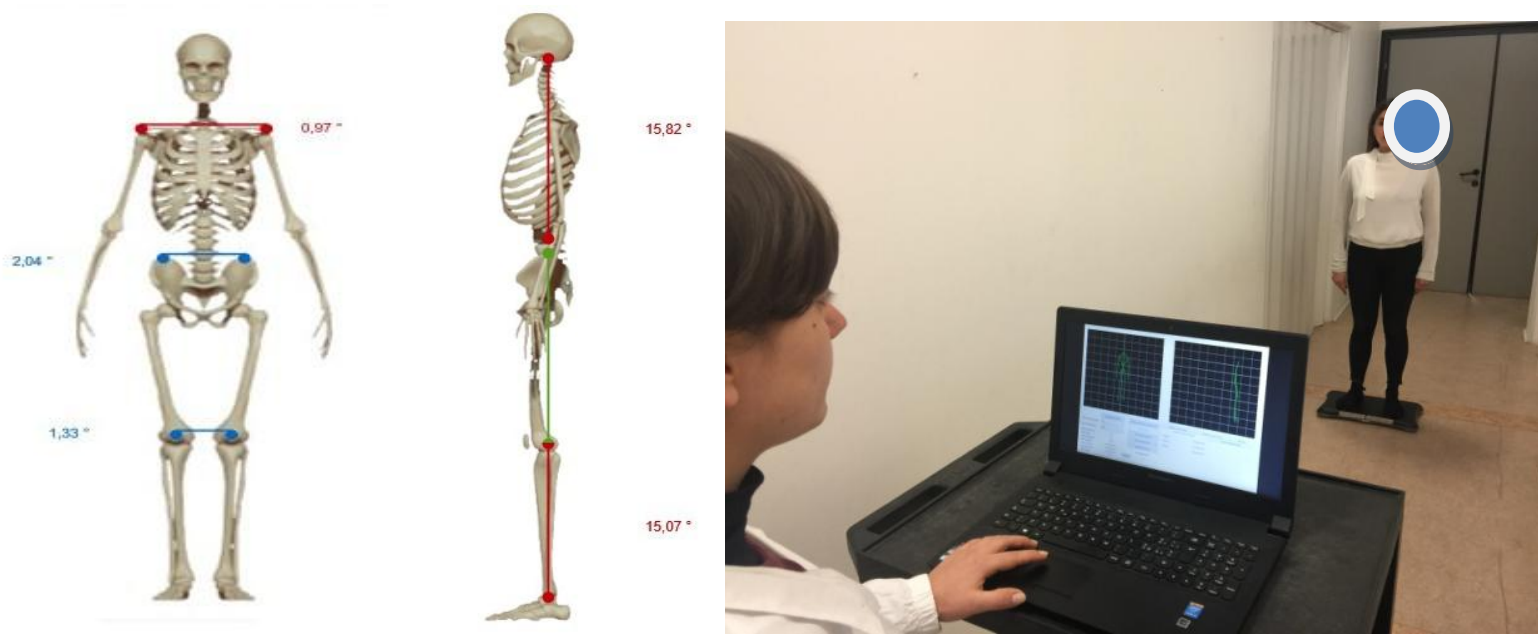

Fig. 1 SaBB Imaging software used as evaluation methods in this study.

\subsection{Treatment procedure}

Each session consisted of 1 hour of treatment. During the session the patient was induced to perform a group of exercises classified in 4 macrocategory:

- $\quad$ Relax and adaptation;

- Myofascial rebalance;

- $\quad$ Correction of the articular range of motion and proprioception;

- $\quad$ Stabilization and strengthening;

These exercises had various grades of complexity and could also be executed with some aids like smile hands, weights, floats, and sticks. They could also be divided into autonomous exercises and assisted exercises, varying on the grade of difficulty and involvement of the physical therapist.
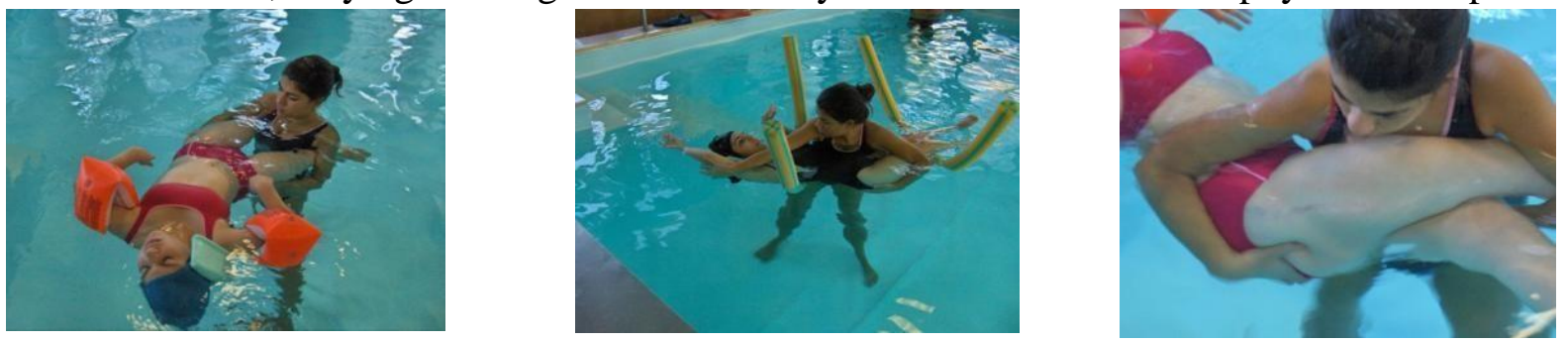

Fig. 2 Examples of exercises used in the Sa.B.B. Technique.

Lastly, each patient was treated with a progressive program of exercises, constantly increasing the amount of work and its complexity at each session, in order to try to obtain a continuous improvement of results by a continuous progressive modification of stimuli administered to the patient.

\section{RESULTS}

Results for 50 subjects (mean age 48.5 years, 23 men and 27 women) were collected and analyzed at the end of the study.

Considering the scores of the FIM scale, neurological patients went from a score of 84,323 to 97,29 points after 20 sessions of treatment $(\mathrm{p}=0.0487)$ while patients with mental disabilities increased from a score of 76,895 to 94,63 points $(p=0.0383$ ) (Graph 1A). In all patients the FIM scale score increased from 82,692 to 95,75 points ( $\mathrm{p}=0.0107)$ (Graph 1B). 

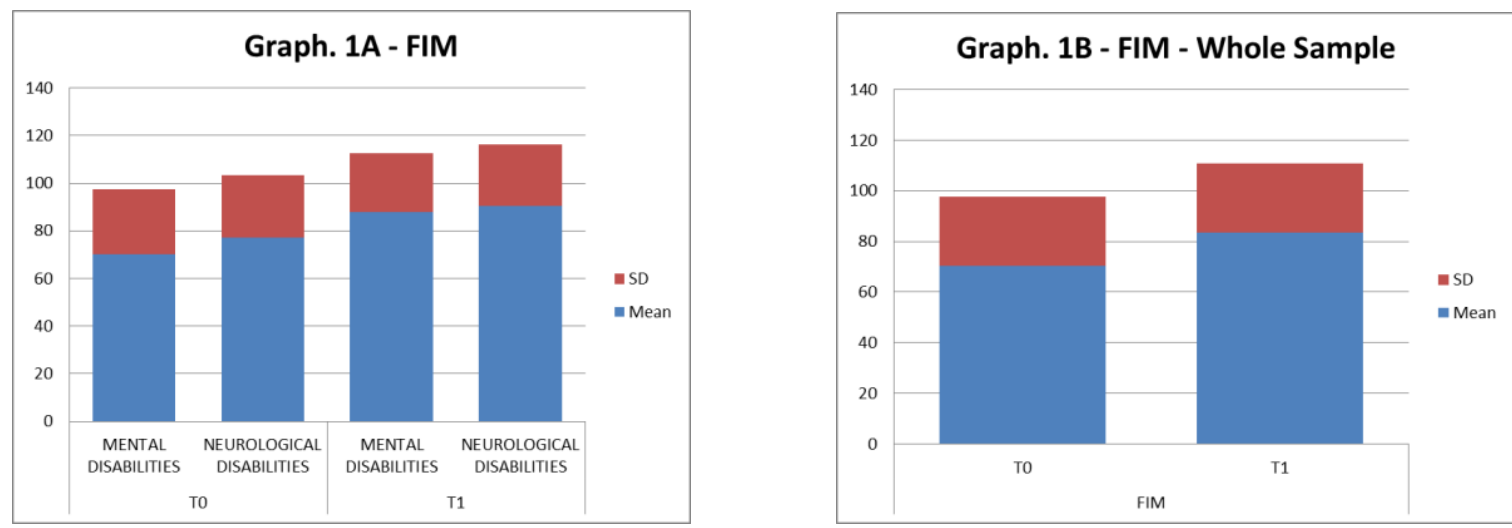

Graph. 1A and 1B Fim scale results at time T0 and T1.

Gait and balance, evaluated through Tinetti scale, improved from 20,105 to 24,474 points $(\mathrm{p}=0.04)$ for patients with mental disabilities and from 11,774 to 13,742 points $(\mathrm{p}=0.4688)$ for patients with neuromotor disabilities (Graph 2A). In all patients the Tinetti score rose from 13,385 to 17,885 points $(\mathrm{p}=0.4688)(\mathrm{Graph} 2 \mathrm{~B})$.
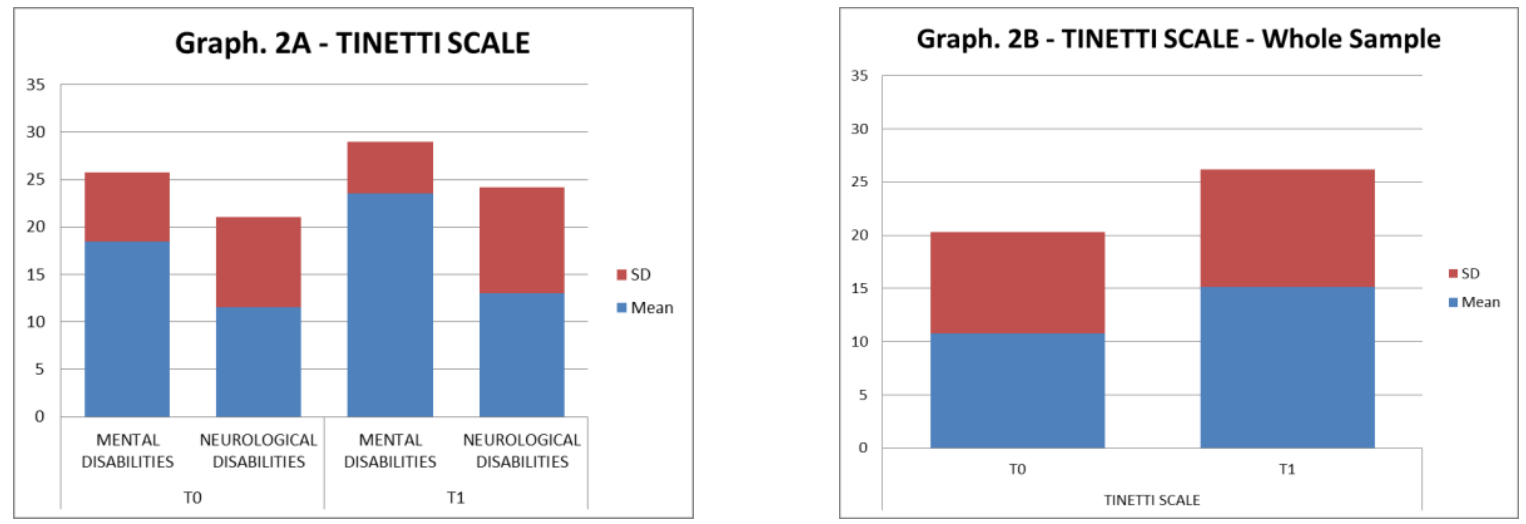

Graph. 2A and 2B Tinetti scale results at time T0 and T1.
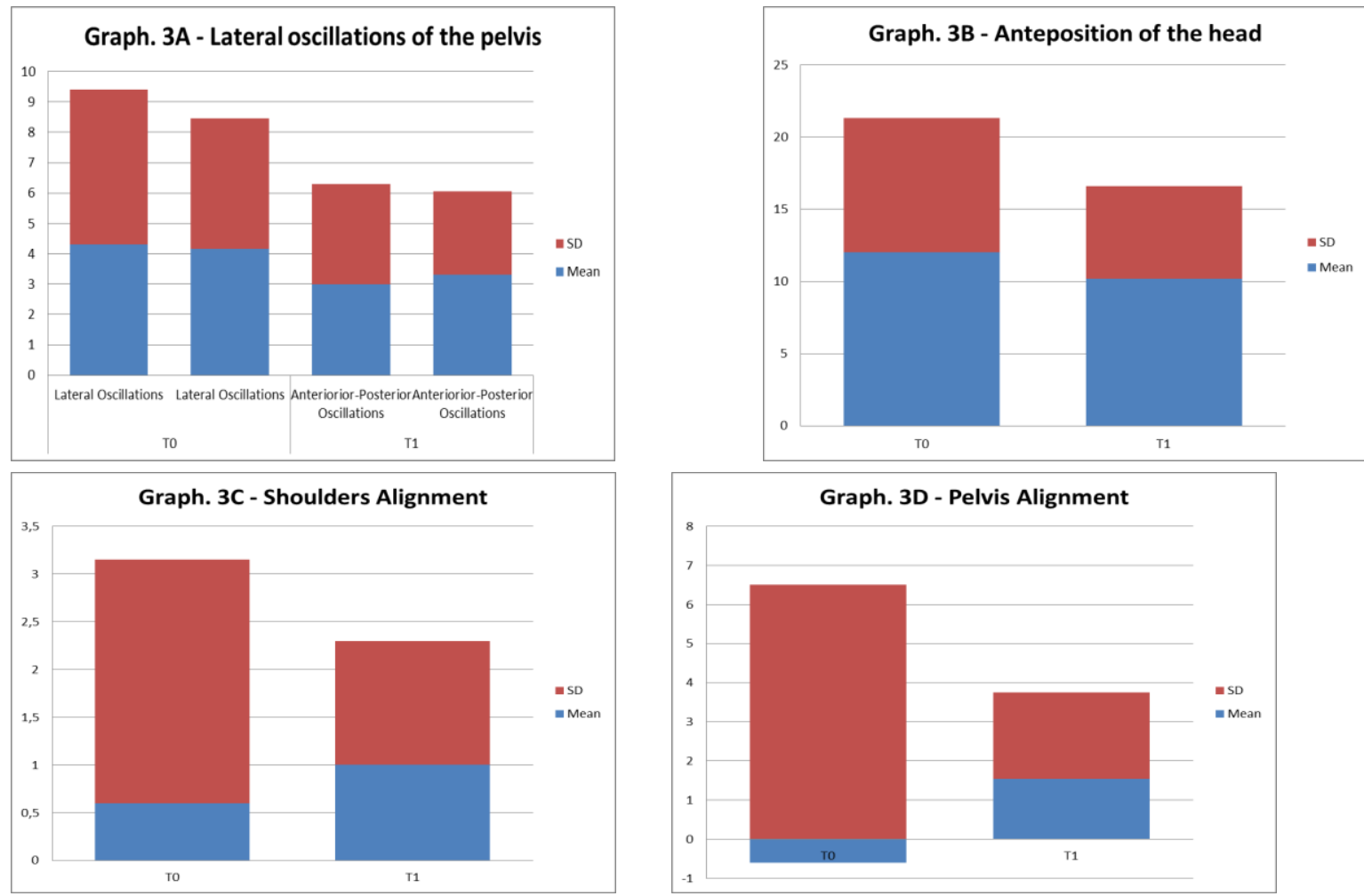

Graph. 3A, 3B, 3C and 3D SaBB Imaging Software results at time T0 and T1. 
These data were validated by the improvements achieved at the postural level, according to the analysis realized with SaBB imaging software, particularly in the values concerning the anteposition of the head, which represents an important parameter for the distribution of the load in the polygon of support (Graph 3B). Furthermore the postural rebalance was objectively supported also by the reduction of the lateral oscillations of the pelvis (Graph 3A).

\section{DISCUSSION}

The result of our study demonstrates how, in the context of a neurological dysfunction inducing static and dynamic postural alterations, the microgravity of the aquatic environment can play an important role in restoring the good balance between audiovisive and proprioceptive inputs perceived by the patient, contributing to its postural rebalance ${ }^{9)}$. This adaptive process is not only limited to the cortical level of elaboration of inputs, but it is also extended to responsive outputs produced by the myofascial system, indicating that it also involves the subcortical, the corticospinal and the spinal level of the nervous system ${ }^{10)}$. In particular, the execution of fine and precise movements, especially in a microgravity environment, has been demonstrated to induce neurogenesis, sinaptogenesis and molecular adaptations in the so stimulated nervous system $^{14), 15), 16)}$. In this view we can substantially postural health as biological luxury ${ }^{11)}$ because it is the complex result of a set of well integrated inputs and outputs that allow the body system to integrate itself with the surrounding environment. We can conclude that the main goal achievable applying specific techniques like the Sa.B.B. is the restoration of this extremely intricate state of healthy "ideal posture" which contributes to the establishment of an intense and long-lasting state of homeostasis of the patient's body's system.

\section{CONCLUSION}

Our study highlighted how the execution of a rehabilitation program in a microgravity aquatic environment using a specific technique combining various kind of exercises and maneuvers could result in a statistically significant improvement of the condition of the patient regarding its general functional balance and posture. These data suggest the necessity to carry out more studies about this kind of approach, in particular by expanding samples used and by having a control group for a better comparison of the outcomes compared to a sham therapy or other kind of rehabilitative approaches.

\section{REFERENCES}

1) Peterka R.J., Loughlin P.J. - "Dynamic regulation of sensorimotor integration in human postural control" J Neurophysiol. - 2004 Jan;91(1):410-23. Epub 2003 Sep 17

2) Travell J. - "Identification of myofascial trigger point syndromes: a case of atypical facial neuralgia" Arch Phys Med Rehabil ? 1981 Mar;62(3):100-6.

3) Travell J., Simons G.D. - "Myofascial pain and dysfunction: the trigger point manual: upper half of body e the lower extremities" - Williams \& Wilkins ? 1992 ISBN-13: 978-0683083668

4) Saggini R., Casini M., Esente S., Panzera F., Sarti G. - "Visione e postura: ginnastica oculare e prescrizione prismatica nel trattamento delle alterazioni posturali" - Fabiano Editore ? 2010

5) Rizk N.N. - "Stereoscopic visualization of various morphological types of collagenous fibres" - Acta Anat (Basel). 1980;107(4):424-9.

6) Wang H.Q., Li M.Q., Wu Z.X., Zhao L. - "The deep fascia in response to leg lengthening with particolar reference to the tension-stress principles" - J Pediatr Orthop. 2007 Jan-Feb;27(1):41-5.

7) Kazmareck K.A., Ostrom N.P., Beebe D.J. - "Microfabricated electrocutaneous tactile display" BMES/EMBS Conference - 1999 10.1109/IEMBS.1999.803993

8) Kazmareck K.A., Nammi K., Agarwal A.K., Tyler M.E., Hasee S.J., Beebe D.J. - "Polarity effect in electrovibration for tactile display" - EEE Trans Biomed Eng. 2006 Oct; 53(10): 2047?2054. 10.1109/TBME.2006.881804

9) Vuillerme N, Nafati G. - "How attentional focus on body sway affects postural control during quiet standing" - Psychol Res ? 2007, Volume 71, Issue 2, pp 192?200 10.1007/s00426-005-0018-2

10) Taube W., Gruber M., Gollhofer, A. - "Spinal and supraspinal adaptations associated with balance 
training and their functional relevance" Acta Physiol (Oxf). 2008 Jun;193(2):101-16 10.1111/j.17481716.2008.01850.x

11) Canguilhem, G. The Normal and the Pathological, Zone Books, 1991, New York.

12) Bellomo R.G., Barassi G. , Pisciella V., Di Pancrazio L. - "Postural reprogramming in the aquatic rehabilitative approach" - Monographies for Updates "Spine and Multidisciplinar Rehabilitation" 4th Evidence-Based Meeting. Pain and Anthalgic Therapies. G.S.S. Milan, 2008, pp. 180-181.

13) Bellomo R.G., Di Pancrazio L.,Pisciella V., Barassi G., Saggini R. - "Articular mobilization treatment and tissues manipulations in aquatic environment in algic-disfunctional patients" - Acts from the XXV national conference of the sport's medicine association of the university G. d'Annunzio of Chieti-Pescara ? 2009

14) Fisher B.E., Petzinger G.M., Nixon K., Hogg E., Bremmer S., Meshul C.K., Jacowek M.W. - "Exerciseinduced behavioral recovery and neuroplasticity in the 1-methyl-4-phenyl-1,2,3,6-tetrahydropyridinelesioned mouse basal ganglia" - J Neurosci Res. - 77(3):378-90 • August 2004 10.1002/jnr.20162

15) Fisher B.E., Wu A.D., Salem G.J., Song J., Lin C.H., Yip J., Cen S., Gordon J., Jacowec M., Petzinger G.M. - "The effect of exercise training in improving motor performance and corticomotor excitability in people with early Parkinson's disease" - Arch Phys Med Rehabil. - 2008 Jul;89(7):1221-9 10.1016/j.apmr.2008.01.013

16) Petzinger G.M., Fisher B.E., McEwen S., Beeler J.A., Walsh J.P., Jakowec M.W.- "Exercise-enhanced neuroplasticity targeting motor and cognitive circuitry in Parkinson's disease" - Lancet Neurol. 2013 Jul; 12(7): 716?726. 10.1016/S1474-4422(13)70123-6 